\title{
Effective antireflection properties of porous silicon nanowires for photovoltaic applications
}

\author{
Adel Najar, Ahmad Ali Al-Jabr, Ahmed Ben Slimane, \\ M. A. Alsunaidi, Tien Khee Ng and Boon S. Ooi \\ Photonics Laboratory, KAUST \\ Thuwal, Kingdom of Saudi Arabia (KSA)
}

\author{
Rachid Sougrat, Dalaver H. Anjum \\ Advanced Nanofabrication, Imaging and Characterization \\ Core Facilities, KAUST \\ Thuwal, Kingdom of Saudi Arabia (KSA)
}

\begin{abstract}
Porous silicon nanowires (PSiNWs) have been prepared by metal-assisted chemical etching method on the n-Si substrate. The presence of nano-pores with pore size ranging between $10-50 \mathrm{~nm}$ in SiNWs was confirmed by electron tomography (ET) in the transmission electron microscope (TEM). The PSiNWs give strong photoluminescence peak at red wavelength. Ultra-low reflectance of $<\mathbf{5 \%}$ span over wavelength $250 \mathrm{~nm}$ to $1050 \mathrm{~nm}$ has been measured. The finite-difference time-domain (FDTD) method has been employed to model the optical reflectance for both $\mathrm{Si}$ wafer and PSiNWs. Our calculation results are in agreement with the measured reflectance from nanowires length of $6 \mu \mathrm{m}$ and $60 \%$ porosity. The low reflectance is attributed to the effective graded index of PSiNWs and enhancement of multiple optical scattering from the pores and nanowires. PSiNW structures with low surface reflectance can potentially serve as an antireflection layer for Sibased photovoltaic devices.
\end{abstract}

Keywords- Porous silicon nanowires; metal electroless etching; FDTD

\section{INTRODUCTION}

Surface antireflection techniques are important for improving the performance of many optical and optoelectronic devices such as solar cells, planar displays and light sensors $[1,2]$. Flat silicon surfaces typically have high spectral dependence surface reflectivity of $35-40 \%$, and limit the total photon flux from entering the depletion region junction of silicon based solar cells. To address this issue, antireflection (AR) layer consisting of multilayer of $\mathrm{SiOx}$, $\mathrm{TiOx}$, or SixNy that form quarter wavelength structures are usually employed [3]. The AR coating method only works effectively in a limited spectral range and for specific angles of incidence [3]. Also, these coatings have several problems such as thermal mismatch, adhesiveness, stability, etc. An alternate approach to minimize reflectivity utilizes a fine surface textured surface, comprising features on the nanometer scale. Silicon nanostructure-based solar cells, such as nanorods, nanocorns, nanotips, and nanowires have attracted much interest because of their novel chemical and physical properties [1,3]. Porous silicon has been intensively studied both experimentally and theoretically due to its photoluminescence and large refractive index [4-8].

Recently, SiNWs arrays have been demonstrated to produce broadband optical absorption because of multiple scattering incidents causing strong light trapping properties [9]. SiNW array has been used as an absorbing layer $[9,10]$. Among the preparation methods for such nanostructures [11, 12], metalassisted chemical etching is well-received as one of the viable technique to produce highly ordered Si nanostructure arrays, as this simple technique is effective and cost effective. Porous silicon nanowires (PSiNWs) prepared by metal-electroless etching technique $(13,14)$ have been widely explored as an alternative top-down route for the fabrication of nanostructures that give visible light emission and good electronic transport properties $[15,16]$.

In this paper, we study the morphology of PSiNWs and the optical properties of this nanostructures. The FDTD simulation was used to fit the measured reflection for Si wafer and PSiNWs for photovoltaic applications.

\section{EXPERIMENTAL DETAILS}

N-type (100) Si wafers with resistivity of 0.01-0.02 $\Omega . c m$ were used in our experiments. The $\mathrm{Si}$ wafers were cleaned using acetone followed by ethanol for 5 minutes in an ultrasonic bath. To remove the organic deposits from the surface, the wafers were soaked in a piranha solution $\left(\mathrm{H}_{2} \mathrm{SO}_{4} / \mathrm{H}_{2} \mathrm{O}_{2}=3 / 1\right)$ for 20 minutes. Silver nanoparticles were then uniformly deposited onto a $\mathrm{Si}$ substrate by reacting $\mathrm{HF}$ (4.8M) with $\mathrm{AgNO}_{3}(0.5 \mathrm{M})$ in deionized water at room temperature for $1 \mathrm{~min}$. The Ag-metal-treated Si wafers were then wet etched in oxidizing hydrofluoric acid solution having $\mathrm{HF}$ and $\mathrm{H}_{2} \mathrm{O}_{2}$ molar concentrations of $4.8 \mathrm{M}$ and $0.4 \mathrm{M}$, respectively. After etching, the samples are treated in $\mathrm{HNO}_{3}$ solution for 15 minutes to completely remove the residual silver particles.

The surface morphology of PSiNWs was investigated using a FEI's Magellan 400 FEG SEM operating at $30 \mathrm{keV}$ beam energy. The crystal structure, size of Si crystallites, and the average pore size were measured using the FEI's TitanG2 80300 TEM equipped with a CCD camera. X-ray energydispersive spectroscopy (EDS) analysis of PSiNWs was performed with an X-ray detector from EDAX attached to the TEM. FEI's Xplore3D software package for the acquisition of electron tomography data from specimens was utilized for performing the 3D-analysis of PSiNWs. Specimens were tilted in the range of $\pm 75^{\circ}$ with an increment of $1^{\circ}$ in order to minimize the distortions appearing the reconstructed volume due to the missing wedge. Finally the reconstructed data was post-processed in Amira package for surface rendering of the PSiNWs.

The photoluminescence (PL) measurements were performed at 
room temperature. A diode-pumped solid-state (DPSS) laser emitting at $473 \mathrm{~nm}$ was used as the PL excitation source. The incident laser power for the measurements was set to $8 \mathrm{~mW}$. The reflectance spectra of the material were studied using MProb UV-Vis spectroscopic system.

\section{RESULTS AND DisCUSSION}

Figure 1 gives SEM micrographs acquired from the crosssection NW samples of the vertically oriented PSiNWs having an average length of $6 \mu \mathrm{m}$ after etching for $45 \mathrm{~min}$. The TEM image of an individual nanowire is shown as inset in Figure 1. The nanowire has a diameter of $\sim 150 \mathrm{~nm}$. The bright-field (BF) TEM micrograph reveals a dense distribution of pores on the surface of the nanowire, with pore sizes varying from 10$50 \mathrm{~nm}$. A Small trace of Ag nanoparticle (AgNPs) has been detected at the bottom part of nanowires using energy dispersive X-ray (EDX). AgNPs have also been observed at the bottom section of NWs (Figure 1 (a))

A typical two dimensional bright-field TEM image used for volume reconstruction of the sample is presented in Figure 2(a), (b) and (c). The three successive longitudinal slices obtained from the reconstructed volume revealed a distribution of irregular size/shape vacuum spaces and silicon frame, constituting the PSiNWs. The shape of the vacuum spaces is similar to these of porous silicon. According to the images, some pores are separated by a distance less than $5 \mathrm{~nm}$. In theoretical prediction of the quantum confinement effect, the characteristic size of the silicon nanocrystallites should be less than the Bohr radius of the free exciton of bulk $\mathrm{Si}$, which is around $5 \mathrm{~nm}$ [17]. Quantum confinement effect is proven by high resolution TEM analysis, in fact it shows small $\mathrm{Si}$ nanocrystallites less than $5 \mathrm{~nm}$ along with the amorphous phase for the sample prepared with 4.8 M HF [15]. P. Kumar et al shows that porous silicon samples with large pore diameter can induce smaller nanocrystalline size of $\mathrm{Si}$ between the pores and lead to a pronounced photoluminescence peak [18].

The PL spectrum of PSiNWs at room temperature is shown in Figure 3 (a). An expected broad band emission was measured, with the pronounced peaks around 670,700 and $760 \mathrm{~nm}$. The $\mathrm{PL}$ emission is attributed to quantum confinement, as explained previously. Also the emission from defect states in surface oxide might contribute to this luminescence $[15,19]$.
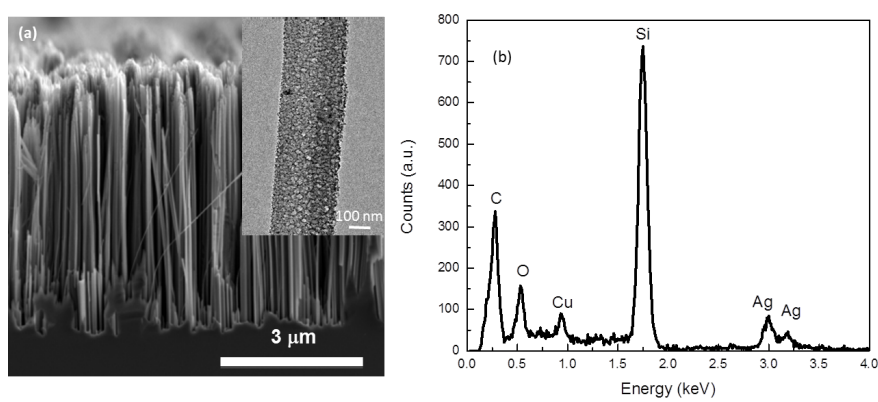

Fig. 1. (a) SEM image of PSiNWs wet etched for 45 min with the inset showing the TEM image of PSiNWs, and (b) EDS spectrum of different elements.
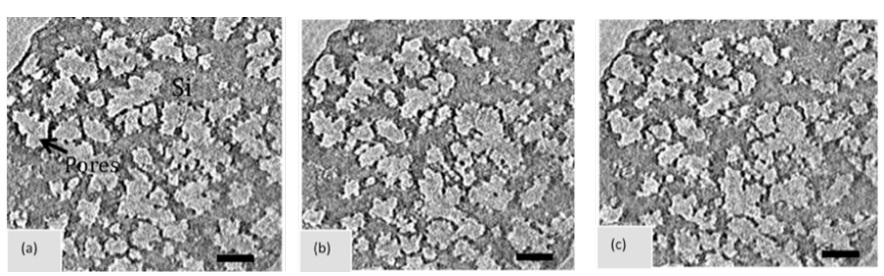

Fig. 2. 2D bright-field TEM image used for volume reconstruction of the sample. (a), (b), and (c) represent three longitudinal slices extracted from the volume reconstruction of the PSiNW nanostructures revealing an area of highly porous medium. The bare scale is $50 \mathrm{~nm}$.

In our case, the aligned structure of Si based PSiNWs could effectively reduce the reflection loss. Optical reflectance spectra of PSiNWs and Si wafer are shown in Figure 3(b). The average reflectance of PSiNWs is less than $5 \%$ over the range of 300-1050 nm much less Si wafer (above 35\%). The very dense PSiNWs with high aspect ratio are vertically aligned, showing a small optical reflection of the incoming light. This remarkably low reflectance of the SiNW array is attributed to several distinct advantages associated with the wire geometry: the ultrahigh surface areas of high-density of PSiNWs; the subwavelength light-trapping effects of PSiNW; and the collective light scattering interactions among PSiNWs and graded refractive index effects, which trap light and make it travel many turns over distances much longer than the array thickness. Consequently, PSiNW solar cells can have extremely low reflectance without any antireflection coatings. In addition, the agglomerated nanowires act as a buffer layer to compensate for the large mismatch in effective index between air and silicon substrate. This excellent anti-reflection property could also be reflected by the dark color of PSiNWs surface. The optical absorption properties of SiNW array films fabricated on glass substrates by metal catalysed electroless etching have been measured by Sivakov et al. and Tsakalakos et al. $[20,21]$.

The SiNW films showed very low reflectance $(<10 \%$ at 300 $800 \mathrm{~nm})$, strong broadband optical absorption

( $>90 \%$ at $500 \mathrm{~nm}$ ), and an optical absorption much higher than Si films with a thickness of $2.7 \mu \mathrm{m}$. The enhanced broadband absorption is attributed to the strong resonance among the aligned SiNW arrays, while the observed
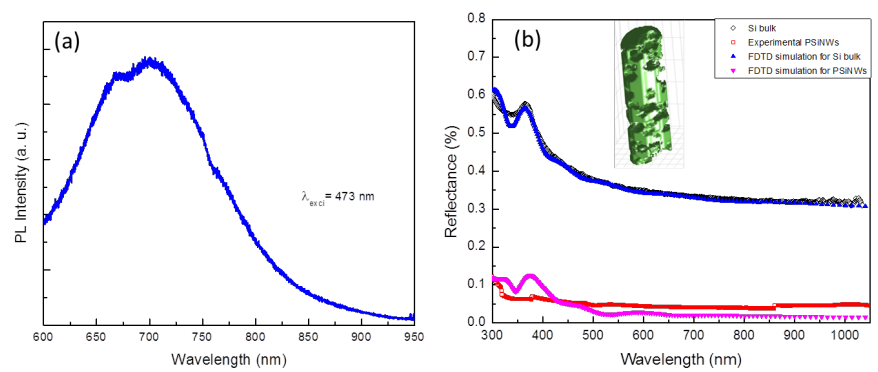

Fig. 3. (a) PL spectrum of PSiNWs with excitation wavelength at $473 \mathrm{~nm}$., and (b) Experimental and FDTD simulated reflectance spectra of Si wafer and PSiNWs. Inset shows the 3D image of individual NW using FDTD simulation. 
absorption is partly due to the high-density surface states in the SiNW film. In our case, the reflectance it's very low compared to SiNWs, so the absorption might higher than SiNWs due to the presence of pores on the nanowires. Garnet and Yang showed that the path length of incident solar radiation of SiNW array films in the AM1.5G spectrum increased by 73 times using optical transmission and photocurrent measurements [22]. The remarkable enhancement factor in the light-trapping path length in PSiNWs might superior to SiNWs and to any traditional lighttrapping method.

The finite-difference time-domain (FDTD) method is used to simulate the optical reflectance of both the $\mathrm{Si}$ wafer and PSiNWs. For the simulation, a 3D FDTD simulator was developed. A uniform plane wave with Gaussian temporal profile is incident on the simulated structure and the reflected fields are recorded in time. The recording points are arranged in a plane parallel to the $\mathrm{Si}$ surface. For each recording location, frequency analysis is performed by Fourier transforming the collected time samples. The results are then normalized to the incident field spectrum and averaged over the whole plane to calculate the overall reflection coefficient. Bulk Si was modeled as a dispersive medium with permittivity $\varepsilon(\omega)$ ranging from $200 \mathrm{~nm}$ to $1100 \mathrm{~nm}$. To model $\varepsilon(\omega)$ for $\mathrm{Si}$, we used the optical properties as given in [23]. The dispersion behavior of $\mathrm{Si}$ in this range was modeled by five Lorentzian terms in (1):

$$
\varepsilon(\omega)=\varepsilon_{0}\left(\varepsilon_{\infty}+\sum_{i=1}^{5}\left(\varepsilon_{\infty}-\varepsilon_{s}\right) \frac{f_{i} \omega_{p i}^{2}}{\left(\omega_{p i}^{2}+2 j \delta_{i} \omega-\omega^{2}\right)}\right.
$$

where $\varepsilon_{-} \infty=1.5$ is the background high-frequency dielectric constant, $\varepsilon_{-} s=3.0$ is the relative static permittivity, $\mathrm{f}_{-} \mathrm{i}$ is the oscillator strength factor, $\omega$ _pi is the plasma frequency in $\mathrm{rad} / \mathrm{s}$ and $\delta_{-} \mathrm{i}$ is the damping factor in $\mathrm{rad} / \mathrm{s}$. A list of these parameters is given in table 1 . To accommodate dispersive response in the FDTD algorithm, the Auxiliary Differential Equation (ADE) approach is used. In particular, the general algorithm based on the generalized polarization formulation recently reported has been implemented [24].

The simulator was tested first for bulk Si. As shown in figure 3 (b), the simulated reflection coefficient matches both the analytical and experimental results. The PSiNWs are modeled in the simulator as a combination of $\mathrm{Si}$ and air. To make the structure of the PSiNWs, the simulator generates air bubbles with random sizes and random locations within the nanowires. The porosity of the PSiNWs is determined by the average percentage of air to $\mathrm{Si}$ in the wires. In this simulation, a periodic structure of nanowires is used with period of 310 $\mathrm{nm}$ and wire diameter of $110 \mathrm{~nm}$. The wires have round tips and length of $6 \mu \mathrm{m}$. A schematic 3D image of a nanowire is shown in the inset of figure 3(b). The Resolution of the simulation is $10 \mathrm{~nm}$ in all directions.
TABLE I.

PERMITTIVITY PARAMETERS FOR THE BULK SI BY CURVE-FITTING

\begin{tabular}{|c|c|c|c|}
\hline Pole & $\mathbf{f}$ & $\boldsymbol{\omega}_{\mathbf{p}}(\mathbf{r a d} / \mathbf{s})$ & $\boldsymbol{\delta}(\mathbf{r a d} / \mathbf{s}) \cdot \boldsymbol{\omega}_{\mathbf{p}}$ \\
\hline 1 & 0.20 & $5.2 \mathrm{e} 15$ & 0.03 \\
\hline 2 & 0.16 & $6.6 \mathrm{e} 15$ & 0.04 \\
\hline 3 & 0.55 & $6.0 \mathrm{e} 15$ & 0.09 \\
\hline 4 & 0.10 & $8.0 \mathrm{e} 15$ & 0.08 \\
\hline 5 & 0.08 & $6.0 \mathrm{e} 15$ & 0.06 \\
\hline
\end{tabular}

As, evident from figure 3(b), The FDTD calculations are in agreement with the measured reflectance for bulk Si wafer as well as PSiNWs with the same parameters as the fabricated sample and with porosity equal to $60 \%$. From these results, PSiNWs are shown to act as an antireflection layer, which eliminates the abrupt change in refractive index between air and silicon substrate, rendering it an effective antireflective layer for photovoltaic devices.

\section{CONCLUSION}

In summary, we fabricated a high density PSiNWs nanostrucutres using metal-assisted chemical etching method. The SEM and 2D-TEM study shows vertically oriented PSiNWs and an irregular size/shape vacuum spaces distribution and silicon frame, constitute the PSiNWs with size pores from 10 to $50 \mathrm{~nm}$. The small distance between pores $(<5 \mathrm{~nm})$ induce very high quantum confinement and lead to a pronounced PL intensity. The fabricated PSiNWs reduce the surface reflection less than $5 \%$ over a broad spectral region, rendering it suitable as an effective antireflection layer. The FDTD simulation is in agreement with the measured reflection for Si wafer and PSiNWs for a length of $6 \mu \mathrm{m}$ and porosity equal to $60 \%$. Using FDTD simulation we will optimize different parameters to improve the manufacturing of the highly ordered PSiNWs for solar cell application.

\section{REFERENCES}

[1] T. Beyer, and M. Tacke: "Antireflection coatings for PbSe diode lasers," Appl. Phys. Lett., vol. 73, pp.1191-1193, 1998.

[2] C.C. Striemer, and P.M. Fauchet, "Dynamic etching of silicon for broadband antireflection applications," Appl. Phys. Lett., vol. 81, pp. 29802982, 2002.

[3] M.A. Green, "Silicon Solar Cells," Advanced Principles and Practice, Bridge Printery, Sydney, 1995.

[4] L. T. Canham, "Silicon quantum wire array fabrication by electrochemical and chemical dissolution of wafers," Appl. Phys. Lett., vol. 57, pp. 1046. 1990.

[5] A. G. Cullis, L. T. Canham, and P. D. J. Calcott, "The structural and luminescence properties of porous silicon,” J. Appl. Phys., vol. 82, pp. 909, 1997.

[6] A. Najar, H. Elhouichet, N. Lorrain, and M. Oueslati, "Excitation mechanisms and localization sites of erbium-doped porous silicon," Appl. Surf. Sci., vol. 252, pp. 5808-5813, 2005.

[7] A. Najar, J. Charrier, H. Ajlani, N. Lorrain, H. Elhouichet, M. Oueslati, and L. Haji, "Optical properties of erbium doped porous silicon waveguides," J. of Lumin., vol. 121, pp. 245-248, 2006.

[8] A. Najar, J. Charrier, N. Lorrain, S. Haesaret, M. Oueslati and L. Haji, "Optical gain measurements in porous silicon planar waveguides codoped by Erbium and Ytterbium ions at $1.53 \mu \mathrm{m}$," Appl. Phys. Lett., vol. 91, pp. 121120, 2007.

[9] M. Lipinski, S. Bastide, P. Panek, and C.L. Clement, "Porous silicon 
antireflection coating by electrochemical and chemical etching for silicon solar cell manufacturing," Phys. Stat. Sol. (a), vol. 197, pp. 512-517, 2003.

[10] M.E. Motamedi, W.H. Southwell, and W.J. Gunning, "Antireflection surfaces in silicon using binary optics technology," Appl. Opt., vol. 31, pp. 4371-4376, 1992.

[11] Y. Cui, L. J. Lauhon, M. S. Gudiksen, J. F. Wang, and C. M. Lieber, "Diameter-controlled synthesis of single-crystal silicon nanowires," Appl. Phys. Lett., vol. 78, pp. 2214, 2001.

[12] J. D. Holmes, K. P. Johnston, R. C. Doty, and B. A. Korgel, "Control of Thickness and Orientation of Solution-Grown Silicon Nanowires," Science, vol. 287, pp. 1471-1473, 2000.

[13] D. Kumar, S. K. Srivastava, P. K. Singh, M. Husain, and V. Kumar, "Fabrication of silicon nanowire arrays based solar cell with improved performance," Solar Energy Materials and Solar Cells, vol. 95, pp. 215-218, 2011.

[14] A. Najar, A. B. Slimane, M. N. Hedhili, D. H. Anjum, T. K. Ng, and B. S. Ooi, "Structural and optical properties of Porous Silicon Nanowires Prepared by Metal-Assisted Electroless Etching Method - Effect of HF concentration," J. Appl. Phys., vol. 112, pp. 033502, 2012.

[15] A. I. Hochbaum, D. Gargas, Y. J. Hwang, and P. D. Yang, "Single Crystalline Mesoporous Silicon Nanowires," Nano Lett., vol. 9, pp 35503554, 2009.

[16] L. W. Sun, H. P. He, C. Liu, Y. F. Lu, and Z. Z. Ye, "Controllable growth and optical properties of $\mathrm{ZnO}$ nanostructures on Si nanowire arrays," Cryst. Eng. Comm., vol. 13, pp. 2439, 2011
[17] Z. G. Bai, D. P. Yu, J. J. Wang, Y. H. Zou, W. Qian, J. S. Fu, S. Q. Feng, J. Xu and L. P.You, "Synthesis and photoluminescence properties of semiconductor nanowires," Mater. Sci. Eng. B, vol. 72, pp. 117-120, 2000. [18] P. Kumar, P. Lemmens, M. Ghosh, F. Ludwig, and M. Schilling, "Effect of $\mathrm{HF}$ Concentration on Physical and Electronic Properties of Electrochemically Formed Nanoporous Silicon," Journal of Nanomaterials, vol. 7, pp. 728957, 2009.

[19] L. H. Lin, X. Z. Sun, R. Tao, Z. C. Li, J. Y. Feng, and Z. J. Zhang, "Photoluminescence origins of the porous silicon nanowire arrays," J. Appl. Phys. vol. 110, pp. 073109, 2011.

[20] V. Sivakov, G. Andr, A. Gawlik, A. Berger, J. Plentz, F. Falk, and S. H. Christiansen, "Silicon Nanowire-Based Solar Cells on Glass: Synthesis, Optical Properties, and Cell Parameters," Nano Lett., vol. 9, pp. 1549, 2009. [21] L. Tsakalakos , J. Balch, J. Fronheiser, B. A. Korevaar, O. Sulima, J. Rand, A. D. Kumar, and U. Rapol, "Strong broadband optical absorption in silicon nanowire films," J. Nanophotonics, vol. 1, pp. 013552, 2007.

[22] E. Garnett, and P. D. Yang, "Light Trapping in Silicon Nanowire Solar Cells," Nano Lett., vol. 10, pp. 1082, 2010.

23] M. A. Green, and M. J. Keevers, "Optical properties of intrinsic silicon at 300 K," Progress in Photovoltaics: Research and Applications, vol. 3, pp.189$192,1995$.

[24] M. A. Alsunaidi, and A. A. Al-Jabr, "A general ADE-FDTD algorithm for the simulation of dispersive structures," Photonics Technology Letters IEEE, vol. 21, pp. 817-819, 2009. 